\title{
Publisher's Note: Extremely large nonsaturating magnetoresistance and ultrahigh mobility due to topological surface states in the metallic $\mathrm{Bi}_{2} \mathrm{Te}_{3}$ topological insulator [Phys. Rev. B 95, 195113 (2017)]
}

\author{
K. Shrestha, M. Chou, D. Graf, H. D. Yang, B. Lorenz, and C. W. Chu \\ (Received 2 June 2017; published 8 June 2017)
}

DOI: 10.1103/PhysRevB.95.239901

This paper was published online on 8 May 2017 with an omission in the Acknowledgments. The Acknowledgments on page 5 should read as "This work is supported in part by the U.S. Air Force Office of Scientific Research, the T. L. L. Temple Foundation, the J. J. and R. Moores Endowment, and the State of Texas through the Texas Center for Superconductivity at the University of Houston. The work at NHMFL is supported by the NSF cooperative Agreement No. DMR-1157490 and the State of Florida. K.S. acknowledges support from the U.S. Department of Energy, Office of Basic Energy Sciences, Materials Sciences, and Engineering Division.” The Acknowledgments have been corrected as of 2 June 2017. The Acknowledgments are correct in the printed version of the journal. 